\title{
Synthesis of Gold Nanoparticles Using Integrated Modular Microfluidic Reactor
}

\author{
Guojun LIU, Wen HONG ${ }^{*}$, Xiaohan PEI, Tengfei WANG, Chunxiu TANG, \\ Shihai LIANG, Feng JIANG, Yanyan ZHANG \\ College of Mechanical Science and Engineering, Jilin University, Changchun 130025, China \\ *mail: Nikkihong@outlook.com
}

Keywords: Piezoelectric actuation; Microfluidic reactor; Synthesis-controlled; Gold nanoparticles

\begin{abstract}
Objective: In order to synthesize the gold nanoparticles with high sphericility, good monodispersity and uniform sizes, a method of synthesizing by integrated modular microfluidic reactor based on piezoelectric actuation is proposed. Method: First, the entrance angle and channel section of the micromixing channel of microreactor are simulated by the software FLUENT, and the related structure parameters are optimized. Second, the two modules of microreactor chips including micromixing chip and growing channel chip are designed, and the microreactor prototype is fabricated. Finally, the synthesis tests of gold nanoparticles based on liquidus chemical reaction are carried out, and the characteristics of the obtained specimen are tested by UV-VIS spectrophotometer and transmission electron microscopy. Result: The test result indicates that with the output flow rate of $4.2 \mathrm{ml} / \mathrm{min}$ and the frequency of $200 \mathrm{~Hz}$, the average particle size and standard deviation of the obtained gold nanoparticles is $(27.89 \pm 5.28) \mathrm{nm}$, and the nanoparticles have uniform sizes, good monodispersity, and high sphericility. Conclusion: The result verifies the feasibility of using the microfluidic reactor presented in this paper to synthesize gold nanoparticles with uniform sizes and good monodispersity.
\end{abstract}

\section{Introduction}

As a new kind of chemical microreactor made by micromachining technologies, microfluidic reactor is widely used in fields like scientific research, biological medicine and chemical engineering because of small preparation dosage [1][2], high safety [3][4], environmental protection [5][6], short reaction time [7][8], etc.

According to different mixing mechanism, microfluidic reactors can be classified into active microreactor and passive microreactor [9]. Disadvantages like poor mixing effect and long mixing time are commonly found in passive microreactor. Accordingly, on the foundation of previous research, a method to synthesize gold nanoparticles by integrated modular active microreactor, which is based on piezoelectric actuation, is proposed. After simulation of the mixing channel, the microreactor is designed and manufactured. Lastly, the advantages and feasibility are proved by the synthesizing-controlled experiment of gold nanoparticles.

\section{Working principle of synthesizing gold nanoparticles by microreactor}

The working principle of synthesizing gold nanoparticles by microreactor proposed in the paper is shown as Fig.1. Micropump A and micropump B, which are totally the same in the structure and function, are used as driving source. When the control signal of the micropump $\mathrm{B}$ is $180^{\circ}$ behind that of the micropump $\mathrm{A}$, the precursor solution $\left(\mathrm{HAuCl}_{4} \cdot 4 \mathrm{H}_{2} \mathrm{O}\right)$ and the reducing agent solution( $\left.\mathrm{Na}_{3} \mathrm{C}_{6} \mathrm{H}_{5} \mathrm{O}_{7}\right)$ will be sent into the mixing channel in a pulsed crossing way. Then, the concentration of gold atoms in the solution keeps increasing, so after the growing channel, the CTAB solution is needed to depolymerize the gathering gold nanoparticles. In the reacting process, gold atoms will gather into crystal nuclei if the concentration of gold atoms exceeds the critical saturation. With the increasing number of crystal nuclei, large amount of gold atoms are consumed and the concentration falls below the critical saturation. Afterwards, the increase of crystal nuclei 
number stops and the crystal nuclei finally grow into gold nanoparticles.

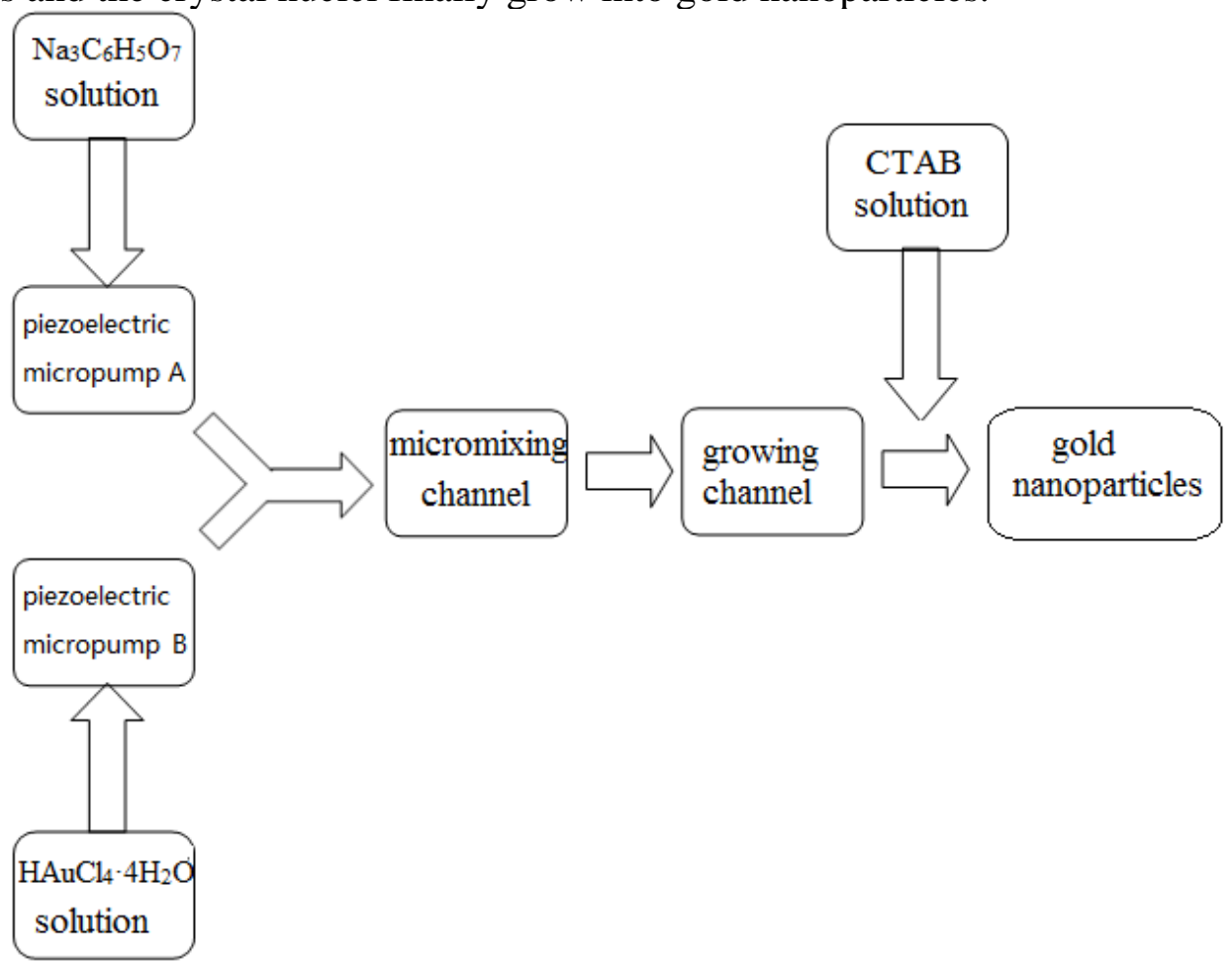

Fig.1 Working principle schematic of microreactor to produce gold nanoparticles

\section{Simulation of mixing channel of the microreactor}

\subsection{Evaluation of mixing efficiency}

To evaluate the mixing efficiency, the mixing degree $(\sigma)$ is proposed to observe the homogeneity of microfluid on certain section of the channel [10]. The parameter $\sigma$ is defined as

$$
\sigma=1.0-\frac{1}{c_{\infty}} \sqrt{\frac{\sum_{i=1}^{N}\left(c_{i}-c_{\infty}\right)^{2}}{N}},
$$

where $N$ is the number of sampling points inside the cross-section; $c_{i}$ is the volume concentration at sampling point $\mathrm{i}$; and $c_{\infty}$ is the ideal volume fraction whose value is 0.5 . The value of $\sigma$ should be between 0 and 1. Larger $\sigma$ indicates better mixing efficiency. When the mixing degree $\sigma=0$, there is no mixture between two phases of fluid; When the mixing degree $\sigma=1$, two phases of fluid are in a real state of complete mixing.

\subsection{Simulation of mixing efficiency with different channel structures}

To study the effect of entrance channel angle on the whole system, the mixing efficiency with entrance channel angle ranging from $30^{\circ}$ to $180^{\circ}$ is analyzed under the condition where the working frequency of micropumps(f) is set as $200 \mathrm{~Hz}$, the output flow rate of micropumps(Q) is set as $4.2 \mathrm{ml} / \mathrm{min}$, and the size of channel section is set as $0.4 \mathrm{~mm} \times 0.4 \mathrm{~mm}$. Then, measure the parameters of the channel sections $X=11, X=12, X=13$ with different angles separately under the simulated condition by the software FLUENT. Finally, output the results into MATLAB and Tab.1 is presented below.

Tab.1 Mixing degree of the test sections of micromixing channel for different angle $\theta$ (The unit of $X$ is $\mathrm{mm}$ )

\begin{tabular}{c|c|c|c|c|c|c}
\hline $\begin{array}{c}\text { Mixing } \\
\text { degree }\end{array}$ & $\theta=30^{\circ}$ & $\theta=60^{\circ}$ & $\theta=90^{\circ}$ & $\theta=120^{\circ}$ & $\theta=150^{\circ}$ & $\theta=180^{\circ}$ \\
\hline$X=11$ & 0.7439 & 0.7604 & 0.7749 & 0.6246 & 0.7789 & 0.8692 \\
\hline$X=12$ & 0.7315 & 0.7409 & 0.8082 & 0.6032 & 0.7682 & 0.8570 \\
\hline$X=13$ & 0.8165 & 0.7145 & 0.8550 & 0.6406 & 0.8201 & 0.8460 \\
\hline
\end{tabular}


It can be found in Tab.1 that the differences of mixing degrees among these three sections are little and the mixing degree reaches highest when $\theta=90^{\circ}$ and $\theta=180^{\circ}$. Considering the integration of the microsystem at the same time, the entrance channel angle is designed as $90^{\circ}$.

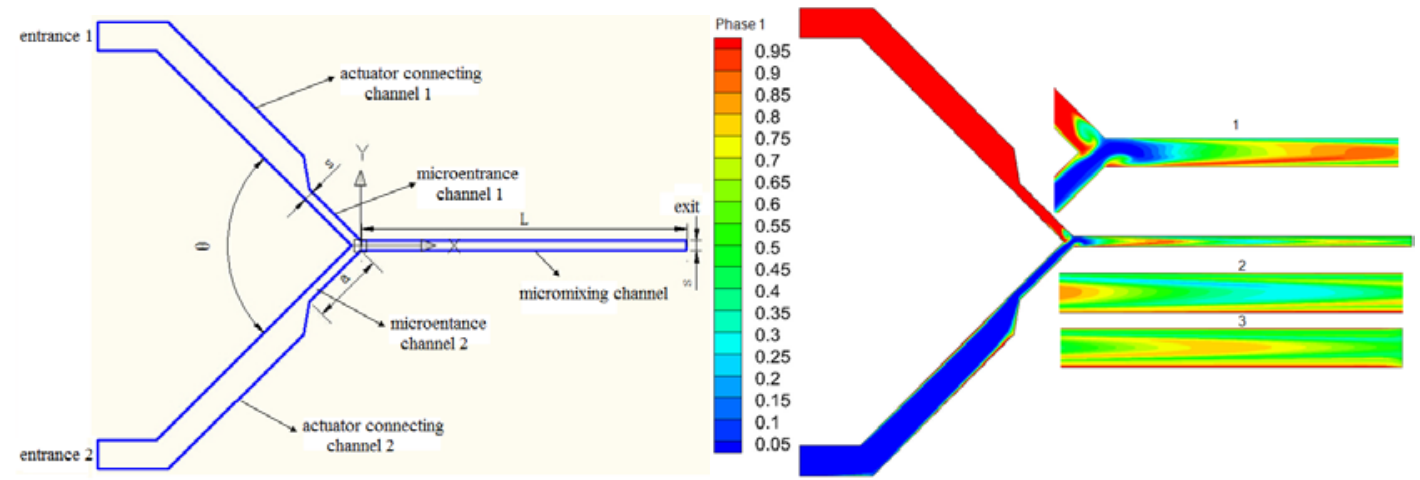

Fig.2 Schematic diagram of 2D geometric structure of micromixing model and simulation

In order to observe the influence of channel section size on mixing efficiency, the mixing efficiency with channel size ranging from 0.2 to $0.5 \mathrm{~mm}$ is analyzed under the condition where the working frequency of micropumps(f) is set as $200 \mathrm{~Hz}$, the output flow rate of micropumps(Q) is set as $4.2 \mathrm{ml} / \mathrm{min}$, and the entrance channel angle is set as $90^{\circ}$. By simulation, the best mixing effect is found when the width of channel is $0.4 \mathrm{~mm}$. In addition, the mixing degree value increases stably from the section $X=7$ when the section size is set as $0.4 \mathrm{~mm}$. That means the mix under such condition takes shorter time and the mixing degree value will be higher than that with other sizes after reaction. Accordingly, the size of channel is set as $0.4 \mathrm{~mm} \times 0.4 \mathrm{~mm}$ in this paper. The mode and the simulation of the micromixing channel are shown inFig.2.

\section{Design and manufacture of the microreactor}

The microfluidic reactor proposed in this paper includes two modules of microreactor chips, namely micromixing chip and growing channel chip. The micromixing chip is designed for the process of mixing accompanied with microreaction. The growing channel chip is for further reaction and some chemical reaction with multiple steps.

\subsection{Design and manufacture of mixing chip}

Piezoelectric pump is the best choice for microfluidic actuator because of easy micromation, low power dissipation and convenient control. The chip is the laminated structure, and from top to bottom are, in order, piezoelectric vibrator, valve 1, valve 2, PDMS substrate, and silicon slice. The PDMS substrate, which is the main part of the micromixing chip, is integrated with entrance, entrance connecting channel, piezoelectric micropump, actuator connecting channel, microchannel, exit, electrode, etc. The piezoelectric vibrator, valve 1 and valve 2 are assembled into the micropump to compose the actuator. Then, seal the substrate on the silicon slice to close the channels and install the electrodes into the electrode bath. The photo of micromixing chip is shown in Fig.3

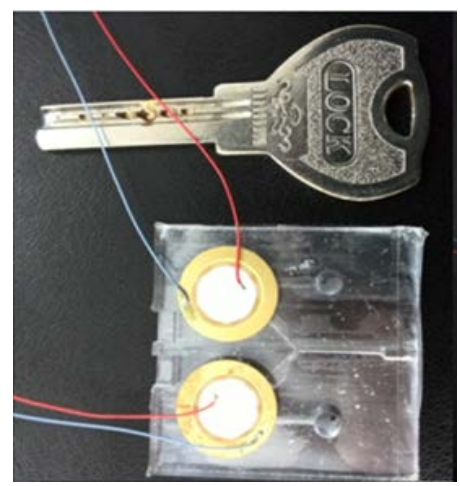

Fig.3 Photo of micromixing chip driven by PZT micropump

\subsection{Design and manufacture of growing chip}

In the growing chip, the entrance, the exit and the growing channel are integrated on the PDMS 
substrate. The channel size is $0.4 \mathrm{~mm} \times 0.4 \mathrm{~mm}$ and the substrate is sealed on the silicon wafer. To avoid side leakage, the exit is designed at the opposite side of the channel, so its external rim is distant from the substrate.

\section{Experiments of synthesizing gold nanoparticle by microreactor}

To prove the feasibility of the microreactor proposed in the paper to produce gold nanoparticles, experiments of synthesizing gold nanoparticles are carried out. The experimental scheme is shown in Fig.4.

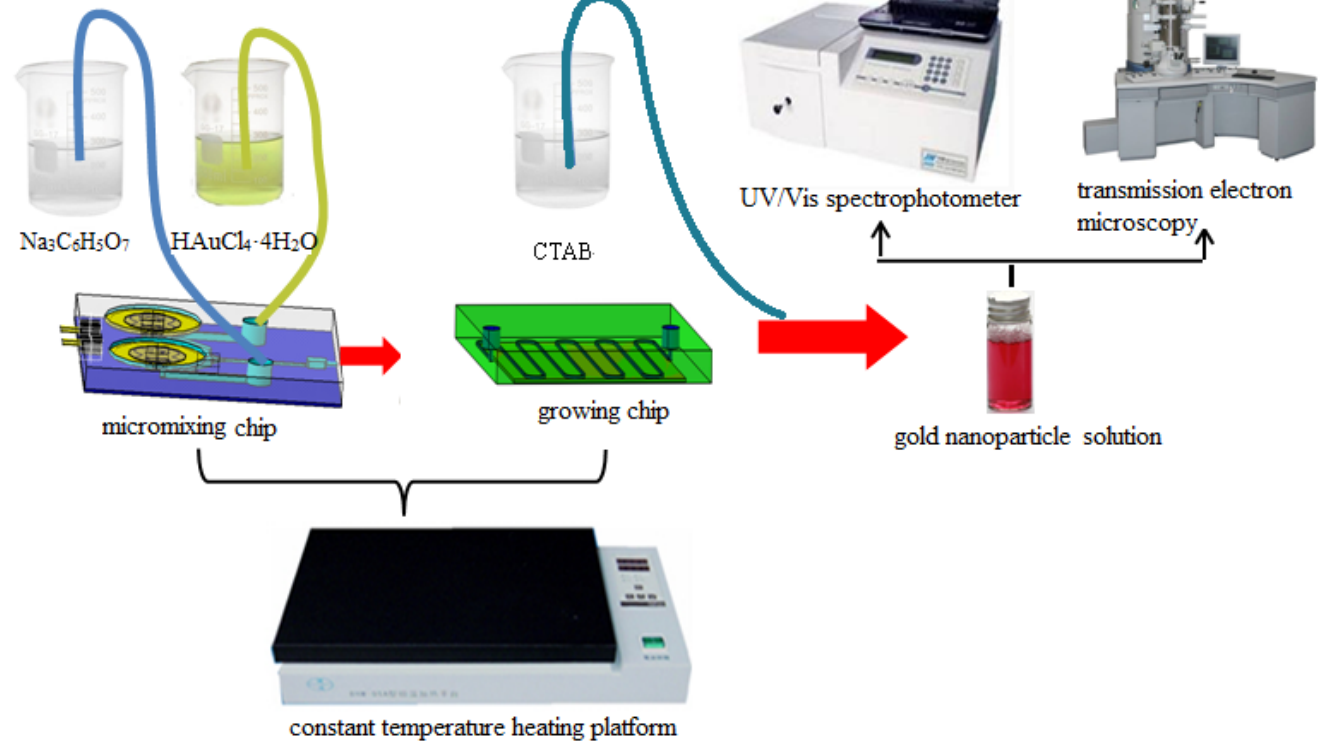

Fig.4 Experimental schematic diagram of controlled synthesis of gold nanoparticles

Reagents and apparatus: $\mathrm{HAuCl}_{4} \cdot 4 \mathrm{H}_{2} \mathrm{O}$ (precursor), $\mathrm{Na}_{3} \mathrm{C}_{6} \mathrm{H}_{5} \mathrm{O}_{7}$ (reducing agent), CTAB, deionized water, UV/Vis spectrophotometer (UV-2501 PC, SHIMADZU, Japan), transmission electron microscopy (TEM TECNAI-12, Philips, Holland), constant temperature heating platform(BHW-05A shanghai, China), power source controller. The temperature of mixing and reaction is set at $100^{\circ} \mathrm{C}$.

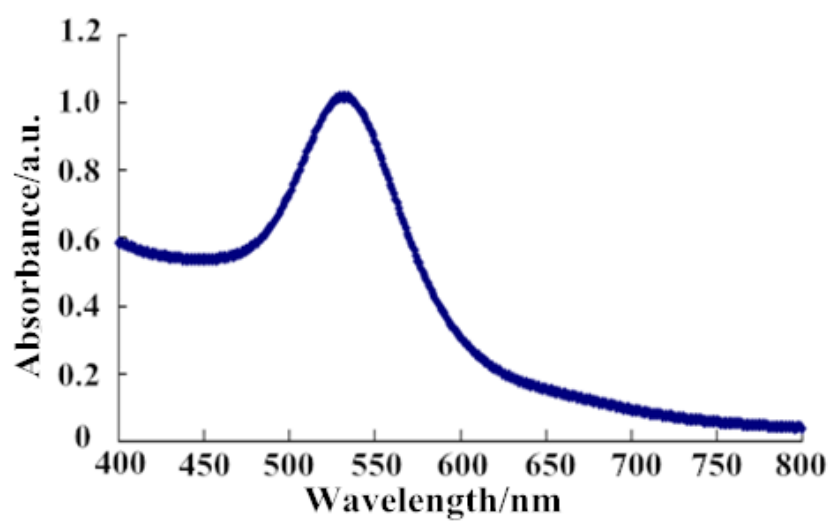

Fig.5 UV-Vis absorption spectra of gold nanoparticles

Fig.5 shows the UV-Vis spectra of gold nanoparticles synthesized with the working frequency of piezoelectric pump as $200 \mathrm{~Hz}$ and the input flow rate as $4.2 \mathrm{ml} / \mathrm{min}$. The maximum absorption wavelength of the solution produced is around $530 \mathrm{~nm}$, which matches the typical and unique optical characteristic of the gold colloid. That means, surface plasmon resonance (SPR) by gold nanoparticles is observed at $500 \sim 550 \mathrm{~nm}$.

Fig.6 shows TEM micrographs and histograms of gold nanoparticles. The average particle size 
and standard deviation of the obtained gold nanoparticles was $(27.89 \pm 5.28) \mathrm{nm}$. It can be seen that the solution presents the wine red and the nanoparticles have good monodispersity and sphericility. The results of the experiments further demonstrate that the microfluidic reactor does work in synthesizing gold nanoparticles .

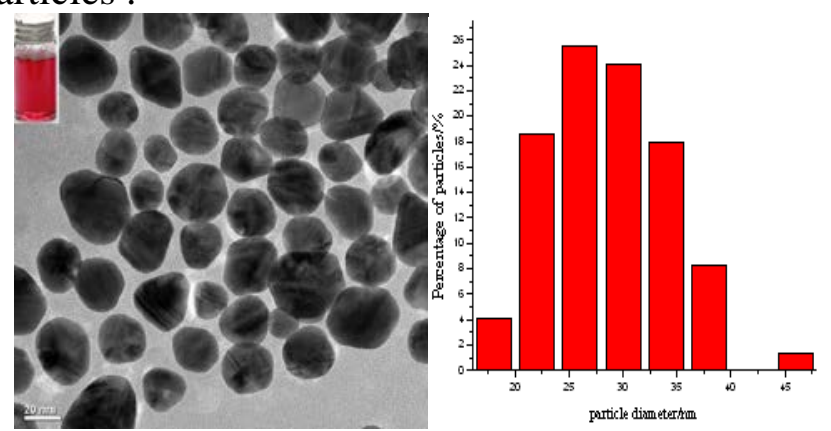

Fig.6 TEM micrographs and histograms of gold nanoparticles

\section{Conclusion}

This paper proposes a new method to synthesize gold nanoparticles by integrated and modularized microfluidic reactor. The parameters are optimized after simulation of microreactor channel and the chips are designed and manufactured. Then the experiments of controllably synthesizing gold nanoparticles are carried out. The results show that the average particle size and standard deviation of the obtained gold nanoparticles is $(27.89 \pm 5.28) \mathrm{nm}$ with the output flow rate of $4.2 \mathrm{ml} / \mathrm{min}$ and the frequency of $200 \mathrm{~Hz}$. The nanoparticles have good monodispersity and sphericility, which verifies the feasibility of integrating piezoelectric micropump with microfluidic reactor and ensures the performance of synthesizing gold nanoparticles. Considering many other influencing factors, subsequent research will study the effect of factors like $\mathrm{pH}$, temperature, stabilizer on controllably synthesizing nanoparticles.

\section{Acknowledgement}

This research is supported and funded by the National Natural Science Foundation Projects (No. 51375207) from Ministry of Science and Technology of the People's Republic of China. Thank the Chain Drive Research Institution of Jilin University for the energetic support and help in the aspect of microdevices fabrication.

\section{References}

[1] GOODELL J R, MCMULLEN J P, ZABORENKO N, et al.. Development of an automated microfluidic reaction platform for multidimensional screening: reaction discovery employing bicyclo[3.2.1]octanoid scaffolds [J]. J. Org. Chem., 2009, 74(16): 6169-6180.

[2] ZABORENKO N, BEDORE M W, JAMISON T F, et al.. Kinetic and scale-up investigations of epoxide aminolysis in microreactors at high temperatures and pressures [J]. Organic Process Research \& Development, 2011, 15(1): 131-139.

[3] LEE C C, SUI G, ELIZAROV, et al.. Multistep synthesis of a radiolabeled imaging probe using integrated microfluidics [J]. Science, 2005, 310: 1793-1796.

[4] PELLETER J, RENAUD F. Facile, Fast and safe process development of nitration and bromination reactions using continuous flow reactors [J]. Organic Process Research \& Development, 2009, 13(4): 698-705.

[5] PIEBER B, KAPPE C O. Direct aerobic oxidation of 2-benzylpyridines in a gas-liquid continuous-flow regime using propylene carbonate as a solvent [J]. Green Chem., 2013, 15: 320-324. 
[6] WILES C, WATTS P, HASWELL S J. Clean and selective oxidation of aromatic alcohols using silica- supported Jones' reagent in a pressure-driven flow reactor [J]. Tetrahedron Letters, 2006, 47: 5261-5264.

[7] HASWELL S J, SULLIVAN B O, STYRING P. Kumada-Corriu reactions in a pressure-driven microflowreactor [J]. Lab on a Chip, 2001, 1: 164-166.

[8] LLLG T, HESSEL V, LÖB P, et al.. Continuous synthesis of tert-butyl peroxypivalate using a single-channel microreactor equipped with orifices as emulsification units [J]. ChemSusChem, 2011, 4: 392-398.

[9] HUANG J, ZHANG J H, WANG S Y. Theory and experimental verification on valveless piezoelectric pump with multistage Y-shape tubes [J]. Opt. Precision Eng., 2013, 21(2): 423-430. (in Chinese)

[10] SHIN S M, KANG I S, CHO Y K. Mixing enhancement by using electrokinetic instability under time- periodic electric field [J]. J. Micromech. Microeng., 2005, 15: 455-462 